\title{
Asymmetric dependencies in perceiving identity and emotion: Experiments with morphed faces
}

\author{
STEFAN R. SCHWEINBERGER, A. MIKE BURTON, and STEPHEN W. KELLY \\ University of Glasgow, Glasgow, Scotland
}

\begin{abstract}
We investigated whether an asymmetric relationship between the perception of identity and emotional expressions in faces (Schweinberger \& Soukup, 1998) may be related to differences in the relative processing speed of identity and expression information. Stimulus faces were morphed across identity within a given emotional expression, or were morphed across emotion within a given identity. In Experiment 1, consistent classifications of these images were demonstrated across a wide range of morphing, with only a relatively narrow category boundary. At the same time, classification reaction times (RTs) reflected the increased perceptual difficulty of the morphed images. In Experiment 2, we investigated the effects of variations in the irrelevant dimension on judgments of faces with respect to a relevant dimension, using a Garner-type speeded classification task. RTs for expression classifications were strongly influenced by irrelevant identity information. In contrast, RTs for identity classifications were unaffected by irrelevant expression information, and this held even for stimuli in which identity was more difficult and slower to discriminate than expression. This suggests that differences in processing speed cannot account for the asymmetric relationship between identity and emotion perception. Theoretical accounts proposing independence of identity and emotion perception are discussed in the light of these findings.
\end{abstract}

People are very effective in perceiving different types of information from faces. Depending on the situation, an observer may be interested in the identity, mood, sex, age, or speech actions of another person, and faces can provide information about all these. Much of the early research on face perception tended to emphasize the stimulus properties of faces, which pose high demands on visual analysis. For instance, this demand on visual analysis was often thought to be the major reason for findings of a superiority of the right hemisphere in face recognition (see, e.g., De Renzi, Faglioni, \& Spinnler, 1968). More recently, however, it has become clear that taskrelated differences in processing, which depend on what information is at the focus of the observer, need to be taken into account in research on face perception (e.g., Sergent, 1985). For instance, whereas a right hemisphere superiority is typically found for the perception of facial identity or expression, a left hemisphere superiority has been reported for lipreading (e.g., Campbell, De Gelder, $\&$ De Haan, 1996; for a review see Ellis, 1989). This sug-

\footnotetext{
The research reported in this paper was supported by the Deutsche Forschungsgemeinschaft through a Heisenberg fellowship and Grant Schw 511/4-1 to S.R.S. We are grateful to Paula Niedenthal, Aude Oliva, and Philippe Schyns for lending us the face stimuli that were used to compute the morphed faces in these experiments. We also thank Andy Young and an anonymous reviewer for helpful comments on an earlier draft of this manuscript. Correspondence should be addressed to $S$. R. Schweinberger, Universität Konstanz, Fachgruppe Psychologie, Postfach 5560, D-78434, Konstanz, Germany (e-mail: stefan.schweinberger@ uni-konstanz.de); after December 15, 1999, S.R.S. will be at the Department of Psychology, University of Glasgow, 58 Hillhead Street, Glasgow G12 8QB, Scotland (e-mail: stefan@psy.gla.ac.uk).
}

gests that these aspects of face perception are mediated, at least to some extent, by different mechanisms.

In one influential model of face perception, Bruce and Young (1986) suggested that the recognition of facial identity, emotion, and facial speech is mediated by functionally independent processes. They suggested, for example, that for identity perception to occur, faces may be "normalized" with respect to emotional expression or facial speech. Yet although this hypothesis of functional independence has received some support from several areas of research, recent findings suggest that there may be conditions in which the different aspects of face perception may interact. We will briefly present the evidence taken to support functional independence first. For example, single unit recordings in the temporal cortex of monkeys have suggested that different cell populations are sensitive to facial identity and emotional expressions, respectively (Hasselmo, Rolls, \& Baylis, 1989). The literature on neuropsychological dissociations further suggests that brain lesions may selectively affect the recognition of either facial identity or emotion, thereby indicating double dissociations between these deficits (e.g., Humphreys, Donnelly, \& Riddoch, 1993). Experiments with positron emission tomography (PET) have also indicated the activation of different brain regions during the perception of facial identity and emotion (Sergent, Ohta, MacDonald, \& Zuck, 1994). Finally, a study by Etcoff (1984) has suggested that when observers are asked to sort cards with images of faces into two piles, they can selectively attend to either identity or expression, without much interference from the irrelevant stimulus dimension. In other experimental studies, when observers were asked to make 
speeded judgments of emotional expression, reaction times (RTs) were not shorter for familiar faces than for unfamiliar faces (Bruce, 1986; Young, McWeeny, Hay, \& Ellis, 1986).

Yet although these studies appear to support the hypothesis of a complete functional independence between the perception of identity, expression, and facial speech, this hypothesis has been challenged in several more recent studies. Walker, Bruce, and O'Malley (1995) have reported that the familiarity of faces modulated the degree to which visual information influenced speech perception in the audiovisual "McGurk" illusion (McGurk \& MacDonald, 1976). Schweinberger and Soukup (1998) have corroborated an influence of face familiarity on speech reading for static photographs of faces; subjects were found to be more efficient in speech reading for personally familiar faces. These authors also investigated effects of variations in an irrelevant stimulus dimension on classifications of faces with respect to a relevant dimension. They used a task originally devised by Garner (1974, 1983 ), in which subjects are asked to classify faces according to either expression or identity. In different conditions, identity and expression could be correlated (e.g., all happy faces showed person $A$ and all sad faces showed person $B$ ), constant (a control condition without variation on the task-irrelevant dimension; e.g., all faces, whether happy or sad, showed person A), or orthogonal (half of the happy and sad faces showed person A and B, respectively). In this task, RTs are predicted to increase in the orthogonal condition, to the extent that the relevant dimension cannot be processed independently of variations in the irrelevant one. The intriguing finding was that when identity was the relevant dimension, RTs were entirely unaffected by irrelevant variations in emotional expression. In contrast, when expression was the relevant dimension, RTs were clearly affected by irrelevant variations in identity. These results suggest an asymmetric relationship, in which identity is perceived independently of expression, but expression perception is influenced by identity.

There is a potential difficulty in the interpretation of Schweinberger and Soukup's (1998) data, however. One might argue that the susceptibility of a relevant dimension to irrelevant variations in another dimension may depend on the relative speed with which both dimensions can be perceived. If identity can be perceived faster than emotional expression, variations in identity might be more likely to affect the perception of emotion than vice versa. After all, if the perception of the "fast" dimension is already completed before the "slow" dimension becomes available, an insensitivity of the "fast" dimension for variations in the "slow" dimension would be unsurprising. Schweinberger and Soukup argued against such an explanation, on the basis of their finding of similar RTs in the control condition for the identity and the emotion classification tasks. Nevertheless, in view of the finding that RTs and error rates were at least slightly-albeit nonsignificantly-higher in the emotion classification task, a more direct investigation of the role of the relative processing speeds of both dimensions seemed appropriate. The major aim of the present study was therefore to replicate the asymmetric relationship between the perception of facial identity and emotion, and to determine to what degree this asymmetry might be related to the relative perceptual difficulty, or processing speeds, of both dimensions.

Noise or spatial frequency filtering are commonly used in order to degrade visual stimuli (e.g., Uttal, Baruch, \& Allen, 1995), especially if the processing speed of visual stimuli is to be varied. However, such a manipulation of face stimuli would probably affect the speeds of emotion processing and identity processing in much the same way. In contrast, we were looking for a manipulation of face stimuli that would allow us to manipulate the speed of either emotion or identity processing more selectively. A relatively recent technique in digital image processing that appears particularly well-suited for a selective manipulation of the perceptual difficulty of facial dimensions in realistic stimuli is morphing. This term refers to a procedure by which a photographic-quality continuum between two images can be created. A typical observation is that most images on a continuum between two identities (e.g., Kennedy and Clinton) are consistently categorized as belonging to one of the two people corresponding to the original stimuli at each end of the continuum. There is only a relatively narrow area of transition in which there is ambiguity as to whether a face shows the one person or the other (see Beale \& Keil, 1995). Similar observations have been reported for the perception of emotional expressions in morphed faces (Calder, Young, Perrett, Etcoff, \& Rowland, 1996; Etcoff \& Magee, 1992; Young et al., 1997). However, Young et al. (1997) also showed that classification RTs increased with increasing distance of an image from the end of the continuum-even when that image was consistently classified as belonging to a particular category. This suggests that even when morphing leaves the consistency of a classification of a given image relatively unaffected, classification RTs faithfully reflect the decreased perceptual salience of these stimuli.

In the present study, we used the morphing procedure in an attempt to manipulate the perceptual difficulty of either identity or expression discriminations selectively in a given set of faces. Starting from four original faces that varied orthogonally on identity (person A, person B) and emotion (happy, angry), we created four different morph continua. Morph continua were created either across identities within a given emotion, or across emotions within a given identity. In Experiment 1, we found that the morphing procedure was indeed able to manipulate the perceptual difficulty in either the identity or the emotion dimension of the stimuli selectively. Experiment 2 was a Garner-type experiment in which the difficulty of the relevant stimulus dimension (relative to the irrelevant dimension) was systematically varied. This allowed us to address the issue of whether the insensitivity of identity 
classifications to irrelevant variations in emotional expression might simply be a result of the greater perceptual speed of the identity dimension in the stimuli.

In Experiment 1, stimuli from eight equidistant points on each of the four morph continua were used, and subjects were asked to classify all of them for either identity (Experiment 1A) or expression (Experiment 1B). This experiment had three aims: First, we wanted to replicate the discontinuous classification functions with continuous stimulus changes that had been recently reported for morphs across facial identities (Beale \& Keil, 1995) or across emotions (Calder, Young, Perrett, et al., 1996; Calder, Young, Rowland, et al., 1996; Young et al., 1997). Second, we wanted to determine whether the morphing process would be able to selectively affect RTs for classifications on the morphed dimension only. Alternatively, one might argue that the morphing process could more generally affect the perceptual difficulty of the stimulifor example, by introducing spurious regions of blur. ${ }^{1}$ Third, given the findings by Young et al. (1997), we expected that classification RTs would increase when images were morphed away from the endpoints even when they were still consistently classified into one category. Therefore, RTs would reflect increased perceptual difficulty, and hence the first experiment should serve to identify those stimuli on the morph continua that showed maximal perceptual difficulty (in terms of classification RTs) while being still classified in a consistent manner. These stimuli were subsequently used in Experiment 2.

\section{EXPERIMENTS 1A-1B}

\section{Method}

Subjects. Eight University of Glasgow undergraduates contributed data to Experiment $1 \mathrm{~A}$, and 8 different undergraduates contributed data to Experiment $1 \mathrm{~B}$.

Stimuli and Apparatus. All stimuli were presented on a Macintosh monitor in gray-scale mode, using the SuperLab software. The bases for the stimulus set were the four original stimuli, displayed in the corners of Figure 1. These stimuli show two different people (top: person A, bottom: person B) displaying two different emotions (happiness and anger). From these stimuli, new photographic-quality stimuli were generated with Morph software, which can produce a continuum between two images. Morph continua were created either from one to the other emotional expression within one identity (horizontal dimension in Figure 1), or from one to the other identity within one emotional expression (vertical dimension in Figure 1).

For each of these four morphs, between 70 and 90 reference points were positioned on each face. These points were located at corresponding landmarks on each of the two original photographs, and the points were then joined. The morphing procedure has two components, warping and fading. Warping basically involves a spatial transformation of the control points from their original position in one image to their final position in the other image. Fading refers to a change in the values of corresponding pixels from the original image to the other image (for details, see, e.g., Beale \& Keil, 1995). For example, a $71: 29$ morph warps all landmark points $29 \%$ of the distance along the line between their initial and final positions, and the values of corresponding pixels are a weighted average, with contributions of $71 \%$ and $29 \%$ of the initial and final images, respectively. ${ }^{2}$
Faces from eight equidistant points on each of the four morphs were stored on disk and used for the experiment (i.e., in proportions of the initial and final images, 100:0, 86:14, 71:29, 57:43, 43:57, $29: 71,14: 86$, and $0: 100$, subsequently termed morph levels). Because the original stimuli were included, this resulted in a total of 28 different stimuli ( 4 morphs with 6 intermediate morph levels each, plus the 4 original stimuli). Figure 1 presents a subset of these stimuli, with morph levels 100:0, 71:29, 29:71, and 0:100.

Procedure. The faces were presented one by one to the subjects, who were instructed to classify each stimulus for either identity as person A or person B (Experiment 1A) or for emotion as "happy" or "angry" (Experiment 1B), using two-choice keypress responses. The subjects were encouraged to respond as fast as possible, provided that they were reasonably confident about their decision. For a given subject, each of the 28 stimuli was repeated 10 times, resulting in a total of 280 trials. The order of stimuli was randomized within each block of 28 . There were short breaks after every 84 trials. Each trial started with the presentation of a fixation point, followed after $500 \mathrm{msec}$ by the stimulus face. Presentation was terminated with a keypress or after $3,000 \mathrm{msec}$; the next trial started $1,000 \mathrm{msec}$ thereafter.

Prior to the experiment, two runs of 16 practice trials were performed in order to familiarize the subjects with the stimuli and the task. The practice trials always involved the four original faces. In the first run of 16 practice trials, these faces were displayed with either a person name ("Peter" or "Simon" for person A or B, respectively) or the emotion label ("angry" or "happy") printed directly below. In the second run of 16 practice trials, the faces were presented without these labels. The experimental trials were initiated only when a subject performed without error on these practice trials, a criterion that happened to be met by all subjects.

\section{Results}

Classification performance. For Experiment $1 \mathrm{~A}$, the percentage of person $B$ responses depending on morph level and facial emotion can be seen in the upper part of Figure 2. With respect to the two morphs that went across the task-irrelevant dimension (i.e., expression), there seems to be no systematic variation in identity classifications. With respect to the two morphs that went across the task-relevant dimension (i.e., identity), it can be seen that except for the most ambiguous morph levels (those with either $57: 43$ or $43: 57$ proportions of either identity), the stimuli were very consistently classified as displaying either person A or person B. These data were subjected to an analysis of variance (ANOVA) with repeated measures on A-to-B morph (8 levels) and facial emotion (2 levels: angry and happy). Epsilon corrections for heterogeneity of covariances, where appropriate, were performed with the Huynh-Feldt method throughout (Huynh \& Feldt, 1976).

The ANOVA revealed a highly significant main effect of morph $[F(7,49)=159.8, p<.001]$, but no significant effect of facial emotion $[F(1,49)<1]$, and no interaction of facial emotion $\times$ morph $[F(1,49)=1.5, p>.20]$. Post hoc analysis using Duncan's multiple range test $(\alpha=$ $.05)$ suggested that this main effect reflected the fact that morph levels $57: 43$ and 43:57 differed significantly both from levels 100:0, 84:16, and $71: 29$, and from levels $29: 71,14: 86$, and 0:100. Differences were not reliable between levels 100:0, 84:16, and 71:29, and between levels $29: 71,14: 86$, and $0: 100$. 


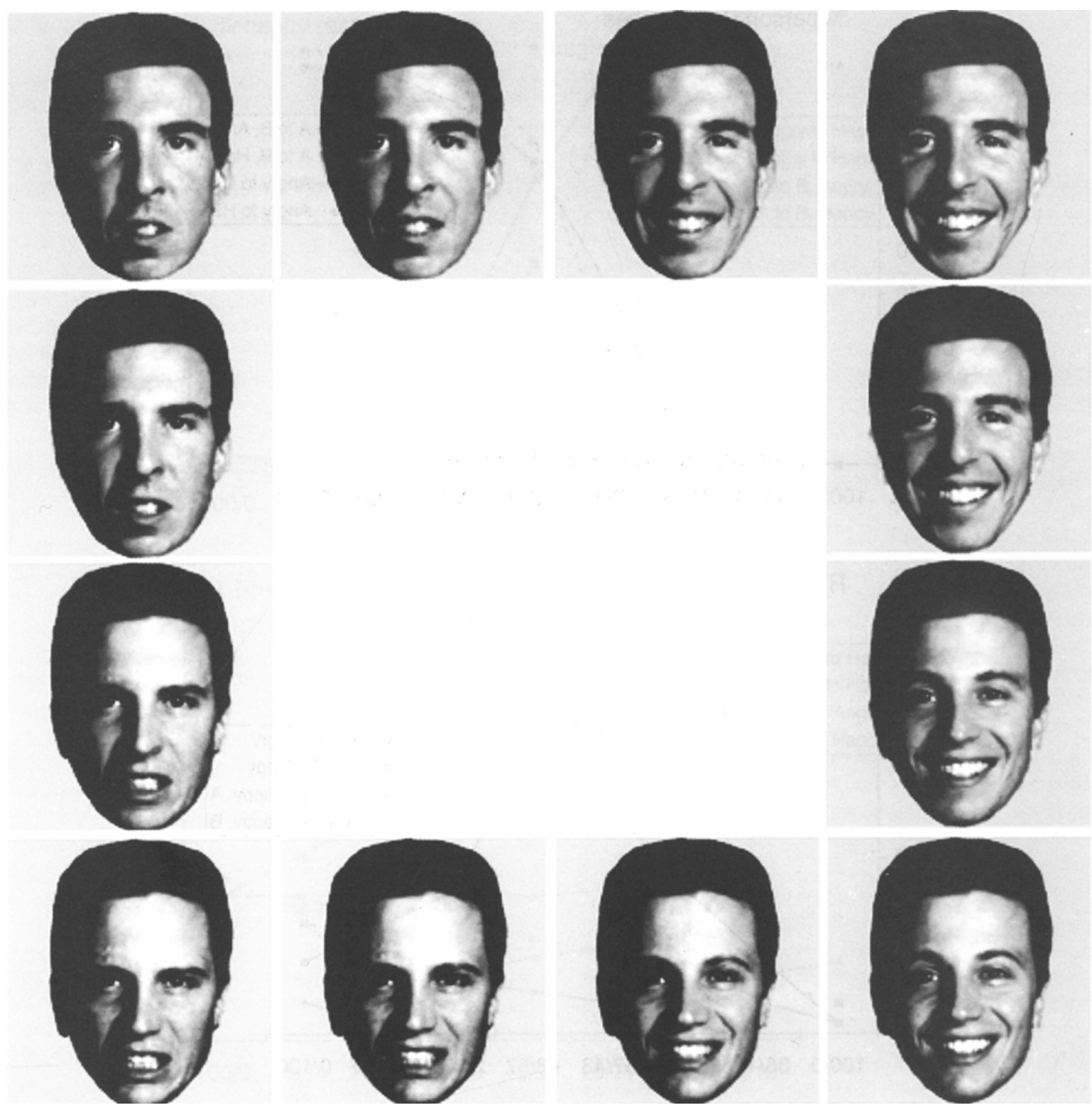

Figure 1. Examples of the stimulus material. The stimuli in the four corners show the original faces. The rows depict a subset of the morphed images from angry to happy emotions within one identity. The columns depict a subset of the morphed images from person $A$ to person $B$ within one emotional expression. The intermediate stimuli represent the $71 \%: 29 \%$ and 29\%:71\% images within each morphed continuum.

For Experiment 1B, the percentage of "happy" responses depending on morph level and facial identity can be seen in the upper part of Figure 3. These data were subjected to an ANOVA with repeated measures on angryto-happy morph ( 8 levels) and facial identity ( 2 levels: A and $B$ ). The main effect of morph was highly significant $[F(7,49)=186.6, p<.001]$. Post hoc analysis using Duncan's multiple range test suggested that this main effect reflected the fact that morph levels $57: 43$ and $43: 57$ differed significantly both from levels 100:0,84:16, and $71: 29$, and from levels 29:71, 14:86, and 0:100. Differences were not reliable between levels 100:0, 84:16, and
71:29, and between levels 29:71, 14:86, and 0:100. In addition, there was a significant effect of facial identity $[F(1,49)=15.1, p<.01]$ and a significant interaction of facial identity $\times$ morph $[F(1,49)=2.7, p<.05]$. Figure 3 suggests that the transition point between angry and happy was slightly earlier for person $A$ than for person $B$ faces.

Classification response times. The classification response times can be seen in the lower parts of Figures 2 and 3 . With respect to the morphs that went across the task-irrelevant dimension, there seems to be no systematic variation in RTs either for identity classifications or 

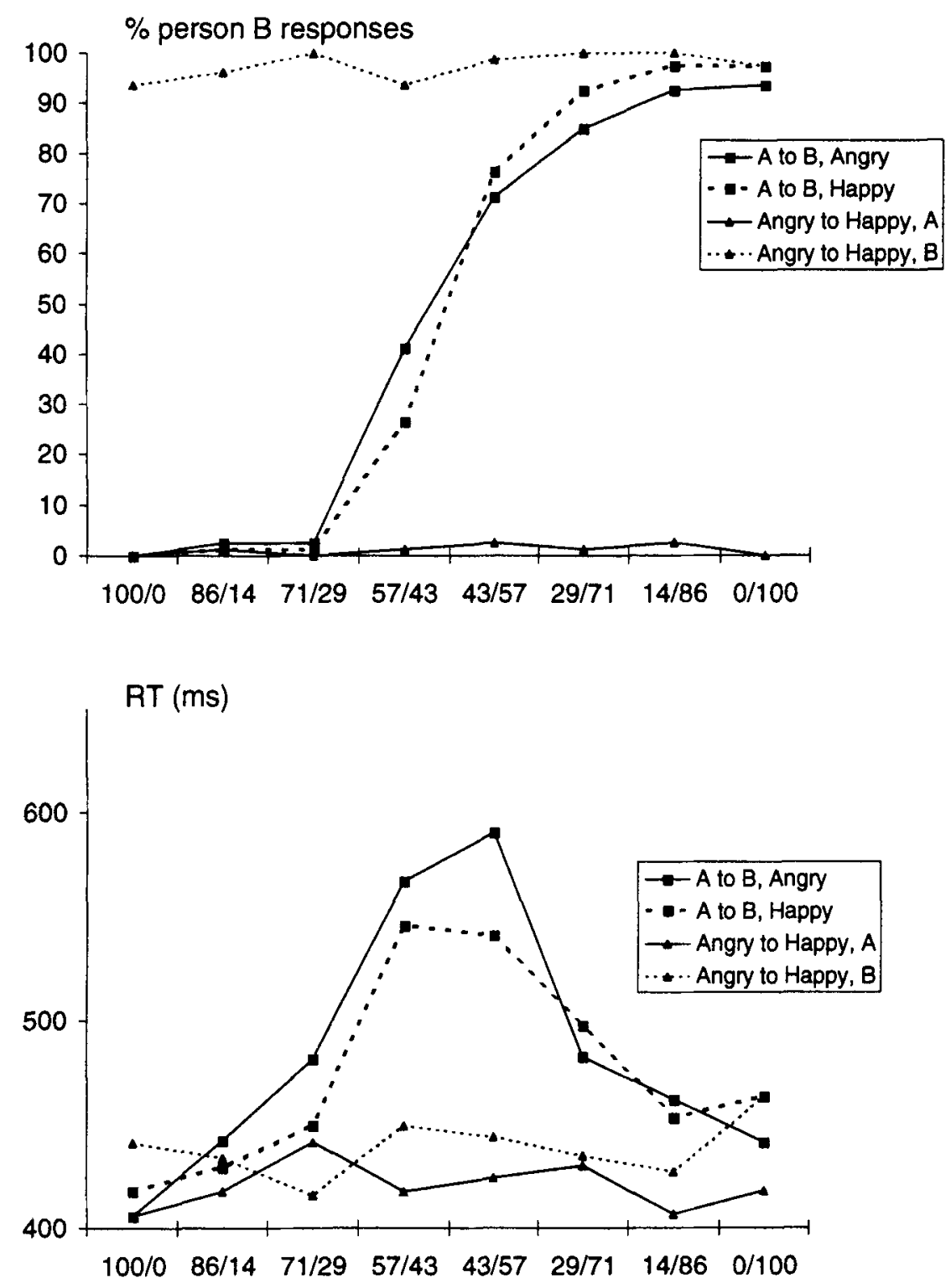

Figure 2. Results of Experiment $1 \mathrm{~A}$ (perception of facial identity). Top: Percentage of "person B" classifications depending on morph level and facial emotion. Bottom: The corresponding classification response times. Square symbols indicate data for the faces morphed across identities within one emotion; triangle symbols indicate data for the faces morphed across emotion within one identity. Note that morphing affects response times only when stimuli are morphed across the target dimension (i.e., across identities).

for expression classifications. With respect to the morphs that went across the task-relevant dimension (i.e., identity in Experiment 1A, and expression in Experiment 1B), Figures 2 and 3 show that RTs were fast for faces close to either end of the morphs, and that RTs were slowest for the most ambiguous stimuli midway between these extremes. Thus, like classification performance, classification speed is most strongly affected in the most ambiguous parts of the morph. Figures 2 and 3 also suggest, however, that RTs showed some sensitivity already for the intermediate morph levels that gave rise to classifi- cations that were similar to those for the original faces (e.g., morph levels 86:14, 71:29, 29:71, and 14:86).

The RT data from Experiment $1 \mathrm{~A}$ were subjected to an ANOVA with repeated measures on A-to-B morph (8 levels) and facial emotion ( 2 levels: angry and happy). The ANOVA revealed a highly significant main effect of morph $[F(7,49)=6.6, p<.01]$, but no significant effect of facial emotion $[F(1,49)=1.01]$ and no interaction of facial emotion $\times$ morph $[F(1,49)<1]$. Post hoc analysis using Duncan's multiple range test suggested that the main effect of morph reflected the fact that morph levels 

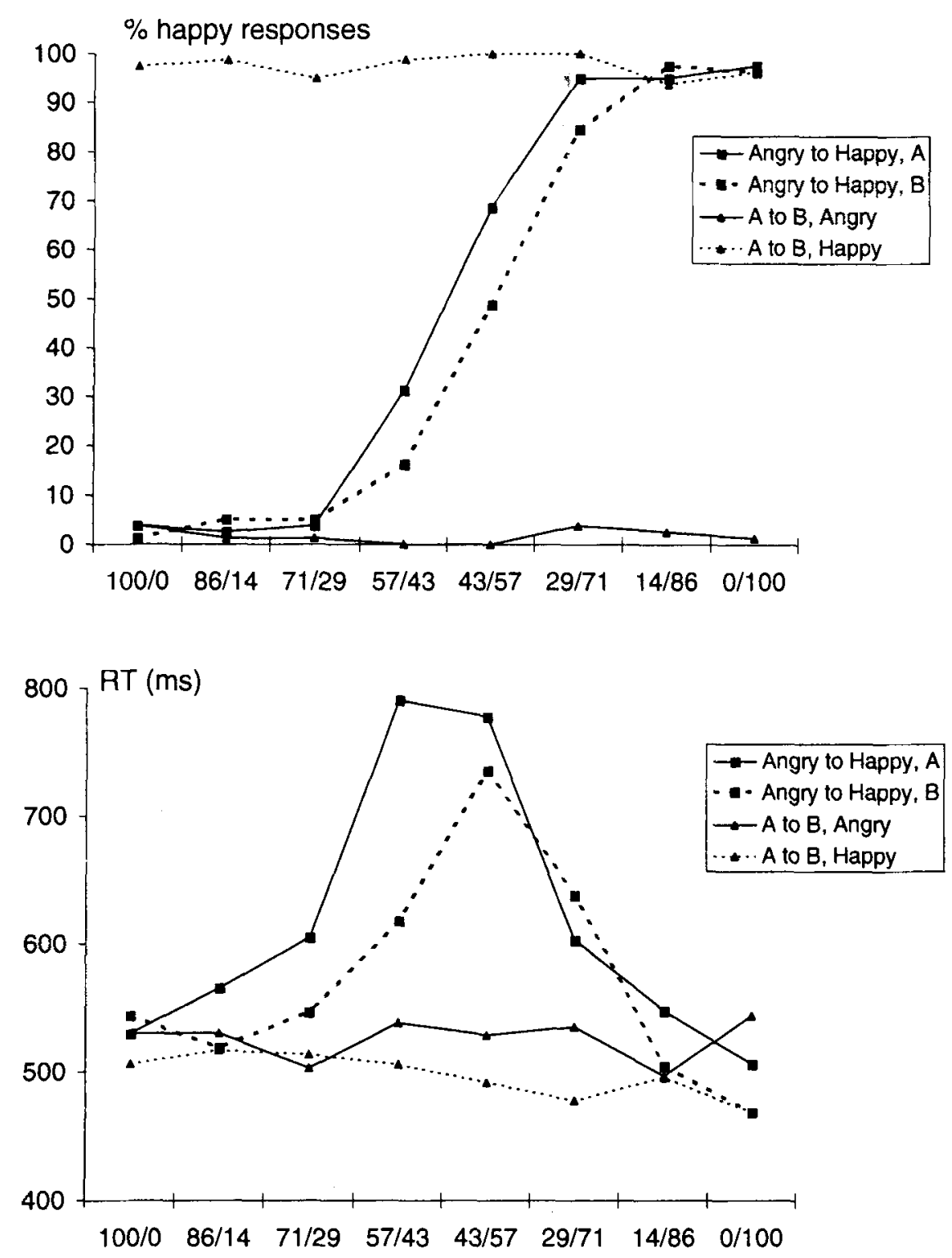

Figure 3. Results of Experiment 1 B (perception of facial emotion). Top: Percentage of "happy" classifications depending on morph level and facial identity. Bottom: Classification response times. Square symbols indicate data for the faces morphed across emotions within one identity; triangle symbols depict the data for the faces morphed across identity within one emotion.

57:43 and 43:57 differed significantly from all other levels except from morph level 29:71.

For Experiment 1B, the ANOVA revealed a highly significant main effect of morph $[F(7,49)=8.6, p<.001]$ and a just significant effect of facial identity $[F(1,49)=$ $5.6, p<.05]$. An inspection of Figure 3 suggests that RTs to person A faces were somewhat longer than RTs to person $B$ faces, but only for the more ambiguous stimuli. However, the interaction of identity $\times$ morph failed to reach significance $[F(1,49)=1.7, p>.20]$. Post hoc analysis using Duncan's multiple range test suggested that the main effect of morph reflected the fact that morph levels $57: 43$ and 43:57 differed significantly from all other levels, with the exception of morph level 29:71.

Inspection of Figures 2 and 3 suggests that, for both experiments, the stimuli on the morph levels $71: 29$ and 29:71 are good examples of perceptually difficult but unambiguous stimuli (at least according to the criterion that they are rather consistently classified into one category, but with slower classification times relative to the original stimuli). To compare RTs directly between the morph levels $100: 0$ and $0: 100$ on the one hand, and the 
morph levels 71:29 and 29:71 on the other hand, we performed additional ANOVAs. There were significant main effects of morph level (100:0 and 0:100 vs. 71/29 and $29 / 71)$, both for Experiment $1 \mathrm{~A}[F(1,7)=17.8, p<.01]$ and for Experiment $1 \mathrm{~B}[F(1,7)=6.6, p<.05]$. In neither experiment were there any further significant effects or interactions with the identity or the emotion of the faces.

\section{Discussion}

These data suggest that when faces on a morphed continuum are presented for classification, there is only a relatively narrow area of transition in which there is ambiguity with respect to whether a face belongs to one or another category. This confirms previous findings obtained for identity (Beale \& Keil, 1995) or emotion classifications (Young et al., 1997). ${ }^{3}$

The RT data show that there is a clear effect on classification speed even for the morph levels for which classifications are very consistent. Therefore, the distance from the original face has a clear cost on the ability to classify the image. This corroborates the findings obtained for facial expressions by Young et al. (1997), and it extends their results to identity classifications.

It might be argued, however, that the costs seen in RTs to the morphed images are not necessarily a result of selectively morphing over either the identity or the expression of a face, but may in part be a consequence of the morphing procedure in general. For instance, longer RTs may be related to a residual loss of stimulus quality in the morphed images. To investigate this possibility, we performed additional analyses that included RTs for the stimuli that were morphed over the dimension that was irrelevant for the task (i.e., when morphed over identity in the expression classification task and vice versa). These ANOVAs included the factors of morph type (i.e., morphed over the task-relevant or the task-irrelevant dimension) and eight morph levels. There was a highly significant interaction of morph type $\times$ morph level in Experiment $1 \mathrm{~A}[F(7,49)=5.8, p<.01]$. A post hoc ANOVA including only the data from the stimuli morphed across the task-irrelevant dimension did not yield any systematic effect on RTs [main effect morph level, $F(7,49)<1$ ], suggesting that morphing along the irrelevant emotion dimension did not affect classification RTs in Experiment $1 \mathrm{~A}$. Similarly, there was a highly significant interaction of morph type $\times$ morph level in Experiment 1B $[F(7,49)=6.6, p<.001]$, and a post hoc ANOVA of only the data from the stimuli morphed across the irrelevant dimension did not yield any systematic effect [main effect morph level, $F(7,49)<1$ ], suggesting that morphing along the irrelevant identity dimension did not affect classification RTs in Experiment 1B. This lack of an effect of morphíng along the irrelevant dimension can also be seen in the lower parts of Figures 2 and 3. It clearly shows that the perceptual effects of morphing can be $s e-$ lective for the stimulus dimension (identity or emotion) across which morphing is performed.

\section{EXPERIMENTS 2A-2B}

In recent experiments (Schweinberger \& Soukup, 1998) with the selective attention paradigm (Garner, 1974, 1983), classifications of facial identity were independent of irrelevant variations in facial emotion, whereas emotion classifications were affected by irrelevant variations in identity. This suggests that observers can attend to identity while ignoring emotional expression. In contrast, observers seem unable to ignore identity when attending to facial emotion.

The aim of Experiment 2 was to determine whether this asymmetric relationship might depend on differences in the relative discriminability of the identity and emotion dimensions. The participants in Experiment 2A classified faces with respect to facial identity, with emotion as the irrelevant dimension. Conversely, the participants in Experiment 2B classified faces with respect to emotion, with identity as the irrelevant dimension. In each experiment, we used as stimuli faces in which the relevant dimension was either easy or difficult to discriminate. The difficult stimuli were always the 71:29 and 29:71 morphed images on the continuum across the relevant stimulus dimension; the easy stimuli were always the endpoints of these continua. In order to maximize the chance for obtaining effects of irrelevant stimulus variations, the irrelevant dimension was always kept easy to discriminate.

These stimuli were presented in three different experimental conditions, termed control, orthogonal, and correlated conditions. In the control condition, the stimuli varied only along the relevant dimension (e.g., identity); the irrelevant dimension (e.g., emotion) was held constant. In the orthogonal condition, stimuli were presented that varied orthogonally along both the relevant and irrelevant conditions. In the correlated condition, there was covariation of the relevant and irrelevant dimensions. For example, there were only happy faces of person A and angry faces of person $B$.

The key question here is how well participants are able to process the relevant dimension independently of variations in the other, irrelevant dimension. The influence of the irrelevant dimension on the processing of the relevant one can be examined in the orthogonal condition. An increase in reaction time for orthogonal as compared with control trials shows that variation along the irrelevant dimension influences classification of the relevant dimension. In other words, exclusive attention to the relevant dimension is impossible; rather, both dimensions are processed in an integral manner (Garner, 1974; Green \& Kuhl, 1991). In contrast, similar RTs for control and orthogonal trials indicate that variation along the irrelevant dimension does not interfere with the perception of the relevant dimension. In this case, the two perceptual dimensions are processed separately.

With respect to the comparison between the control and the correlated condition, a "redundancy gain" (i.e., faster RTs in the correlated condition) is also usually consid- 
ered as an indication of integral processing, indicating that at some level the combination of features is perceived as a unitary event (e.g., Etcoff, 1984; Garner, 1974). However, it has been pointed out that a redundancy gain could also occur in the context of parallel but independent processing of both dimensions: If perceivers classify the stimuli in the correlated condition by systematically using the faster or more discriminable dimension for each trial (in spite of the task instruction), this could also result in a redundancy gain (Eimas, Tartter, Miller, \& Keuthen, 1978; Green \& Kuhl, 1991). Consequently, a redundancy gain should not in general be considered as a strong indication of integral processing.

The main focus in Experiment 2 is therefore clearly on the comparison between control and orthogonal trials. In these conditions, subjects must rely on the relevant dimension in order to solve the task. In the correlated condition, although the instruction is to attend to the relevant dimension, subjects might utilize either dimension for their classifications. We might expect some degree of redundancy gain in Experiment 2 simply because the design was such that the relevant dimension was more difficult to discriminate, on the average, than the irrelevant dimension (see above). Nevertheless, in interpreting the performance in the correlated trials, one should consider the effects of our manipulation in discriminability of the relevant dimension relative to the irrelevant dimension: One would expect a clear effect of difficulty in the control condition, where only the relevant dimension can be utilized for classification. However, to the extent that the more discriminable irrelevant dimension is used for the classification in the correlated condition, one would predict that the effect of difficulty is reduced in that condition.

\section{Method}

Subjects. Twelve University of Glasgow undergraduates contributed data to Experiment $2 \mathrm{~A}$, and 12 different undergraduates contributed data to Experiment 2B.

Stimuli. The stimuli were a subset of those used in Experiment 1. As easy stimuli, we used the four original faces. As difficult stimuli, we selected the 71:29 and 29:71 morphed faces, which according to Experiment 1 were more difficult to discriminate relative to the original faces (as measured by classification RTs) while still being consistently classified into one category. The stimuli in Experiment $2 \mathrm{~A}$ that required identity classifications were those in the left- and rightmost columns of Figure 1. Conversely, the stimuli in Experiment $2 \mathrm{~B}$ that required emotion classifications were those in the top and bottom rows of Figure 1.

Procedure. The subjects were required to make speeded twochoice classification judgments of four types of stimuli that varied along the two dimensions of identity (person A, person B) and emotional expression (angry, happy). The instructions emphasized both speed and accuracy. The participants in Experiment 2A were instructed to classify the faces only along identity - to press one key for faces of person A and another key for faces of person B-while disregarding emotional expression. The subjects in Experiment 2B were instructed to classify the stimuli only along expression--to press one key for happy faces and another key for angry faceswhile disregarding identity.

In each of the three experimental conditions (correlated, control, orthogonal), two consecutive blocks of 136 trials each were pre- sented. Each of these blocks consisted of 16 practice trials, followed without interruption by 120 test trials. In the orthogonal condition, both blocks of test trials consisted of all four possible combinations of the relevant and irrelevant dimension. In the control condition, the particular alternative of the irrelevant dimension was changed between the two consecutive blocks (e.g., first block, only happy faces of persons $A$ and $B$; second block, only angry faces of persons $A$ and $B$ ). In the correlated condition, the particular combination of the relevant and the irrelevant dimensions was changed between the two consecutive blocks (e.g., first block, person A looking happy and person $B$ looking angry; second block, person A looking angry and person $B$ looking happy). This was done to ensure that all stimuli appeared with equal frequency in all experimental conditions, in order to eliminate the possibility that differences among conditions were due to stimulus differences. Within each block, all test trials were presented in a different randomized order with the restriction that every permitted combination of relevant and irrelevant dimensions appear with equal frequency. The order of the experimental conditions was counterbalanced across participants. Breaks were allowed after each block. Each experimental session lasted for about $40 \mathrm{~min}$.

Each trial started with the presentation of a fixation cross, which was replaced after $1,500 \mathrm{msec}$ by a stimulus face. The face remained visible until a response key was pressed and was then immediately replaced by the fixation cross that marked the beginning of the next trial. The participants were to press one key for one alternative of the relevant dimension (e.g., person A) and the other key for the other alternative (e.g., person B). Errors were indicated by a brief acoustic signal. The participants used the left index finger for responses to person A or happy faces, and the right index finger for responses to person B or angry faces.

Responses were scored as correct if the appropriate key was pressed within a time window lasting from 150 to $2,000 \mathrm{msec}$ after target onset. Errors of commission (wrong key) were recorded separately. RTs more than 2 SDs below or above the mean RT for an individual subject and condition were considered as outliers and discarded. Mean RTs were calculated for correct responses to test trials only.

For each experiment, ANOVAs were performed on the test trial RTs, with repeated measures on the variable condition (correlated, control, orthogonal). Additional variables were the particular alternative of the relevant dimension (depending on the experiment, e.g., stimulus person $A$ or $B$ ) and the particular alternative of the irrelevant dimension (depending on the experiment, e.g., happy or angry expression). A comparison between experiments was also performed, using an ANOVA with repeated measures on condition and experiment as a between-subjects variable. We did not use the particular alternatives of the relevant and irrelevant dimension as variables for this between-experiment comparison, because the levels of these variables were obviously not comparable for Experiments $2 \mathrm{~A}$ and $2 \mathrm{~B}$.

\section{Results}

Error rates were small, with an average percentage of errors of commission of $2.9 \%$ and $2.7 \%$ in Experiments $2 \mathrm{~A}$ and $2 \mathrm{~B}$, respectively. Although error rates showed some influence of the experimental conditions, the pattern of error rates was generally similar to that observed in RTs, in both Experiment $2 \mathrm{~A}[M($ easy) $=1.6 \%, 1.3 \%$, and $1.5 \%$, for the correlated, control, and orthogonal condition, respectively; $M$ (difficult) $=2.8 \%, 5.3 \%$, and $4.7 \%]$ and Experiment $2 \mathrm{~B}[M$ (easy) $=1.7 \%, 2.1 \%$, and $2.2 \% ; M$ (difficult $)=2.2 \%, 3.3 \%$, and $4.9 \%]$. Error rates were not analyzed further.

Inspection of Figures 4 and 5 suggests that in general, RTs were slower for the difficult than for easy stimuli, 
Experiment 2A: Identity Easy

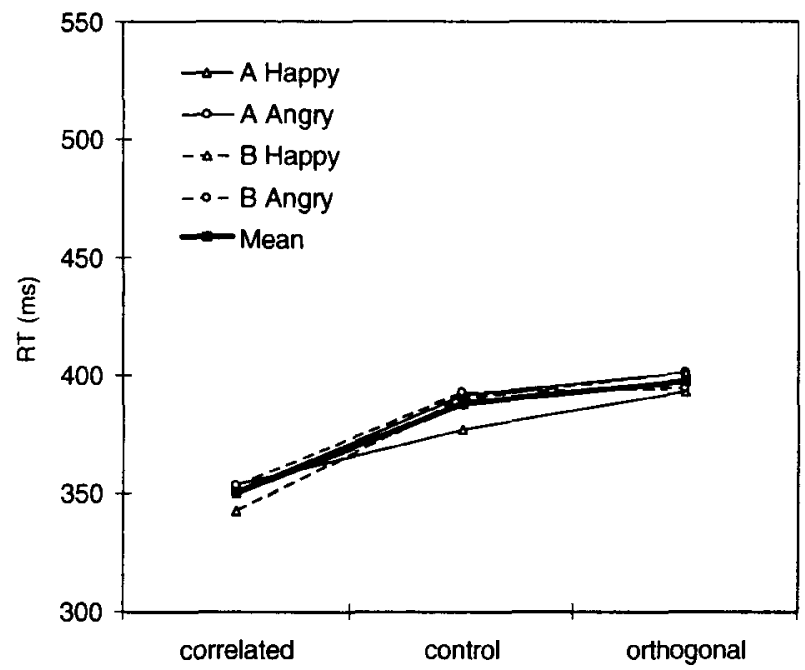

Experimental Condition

\section{Experiment 2B: Emotion Easy}

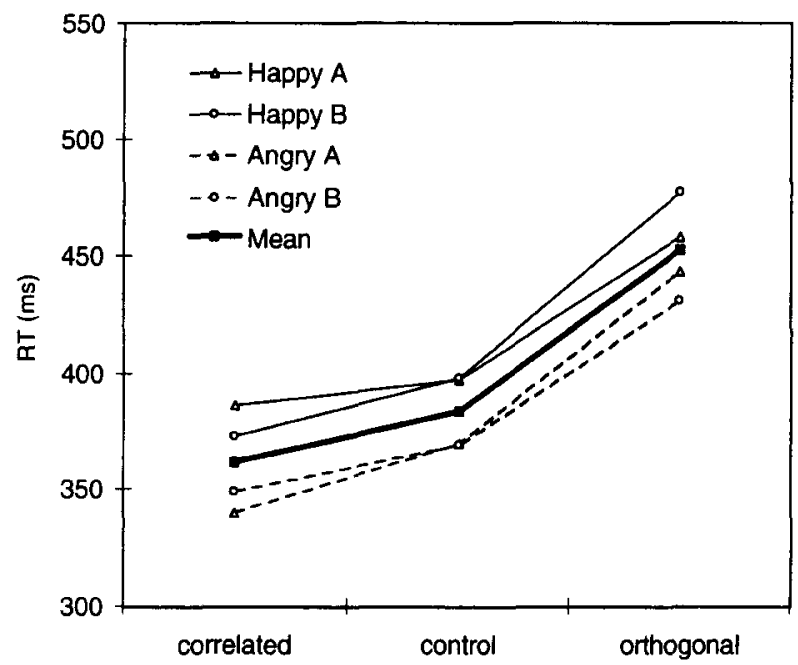

Experimental Condition
Experiment 2A: Identity Difficult

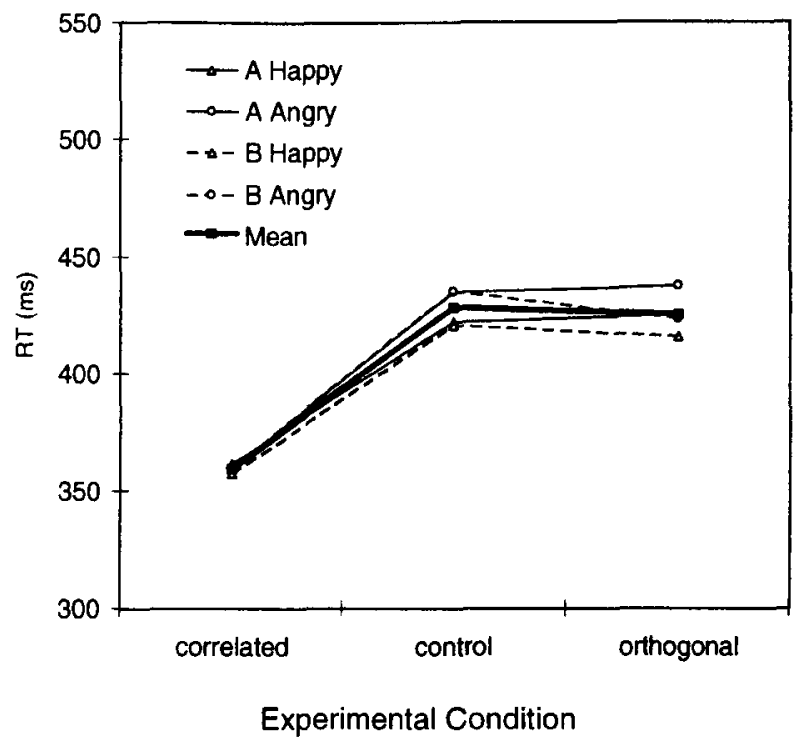

Experiment 2B: Emotion Difficult

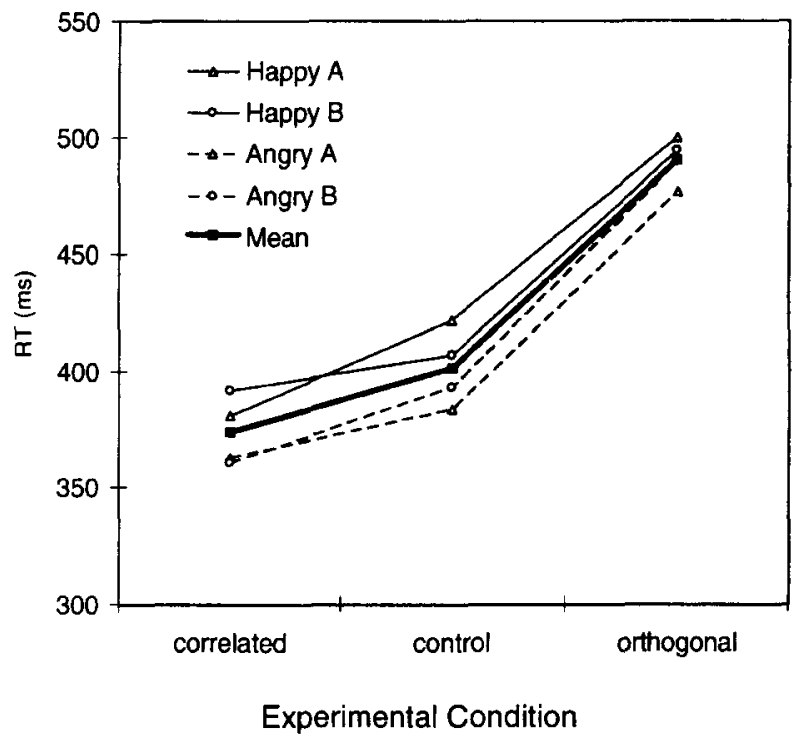

Figure 4. Mean reaction times in Experiments $2 A$ (top) and 2B (bottom) for the experimental conditions and every combination of relevant and irrelevant dimensions. Bold lines show means across all combinations of relevant and irrelevant dimensions.

and that RTs increased over the experimental conditions. However, although there was some degree of redundancy gain in both experiments, there were striking differences between the experiments in terms of the effects of the orthogonal condition: Irrelevant variations in identity interfered strongly with classifications of emotional expression, but irrelevant variations in emotional expression did not seem to interfere at all with classifications of identity. The insensitivity of identity classifications for expression variations was upheld even for the difficult stimuli, in which the relevant identity dimension was actually less salient (in terms of classification RTs in the control condition; see Figure 5) than the irrelevant expression dimension.

These impressions were verified by the statistical analyses. We initially performed an ANOVA across experiments, with repeated measures on the variables of condition (correlated, control, orthogonal) and difficulty (easy, difficult) and the between-subjects variable of experiment. This ANOVA revealed no main effect of experiment $[F(1,22)=1.09, p>.20]$, indicating that the RTs during the classification for identity were similar to those during the classification for emotion. However, there 


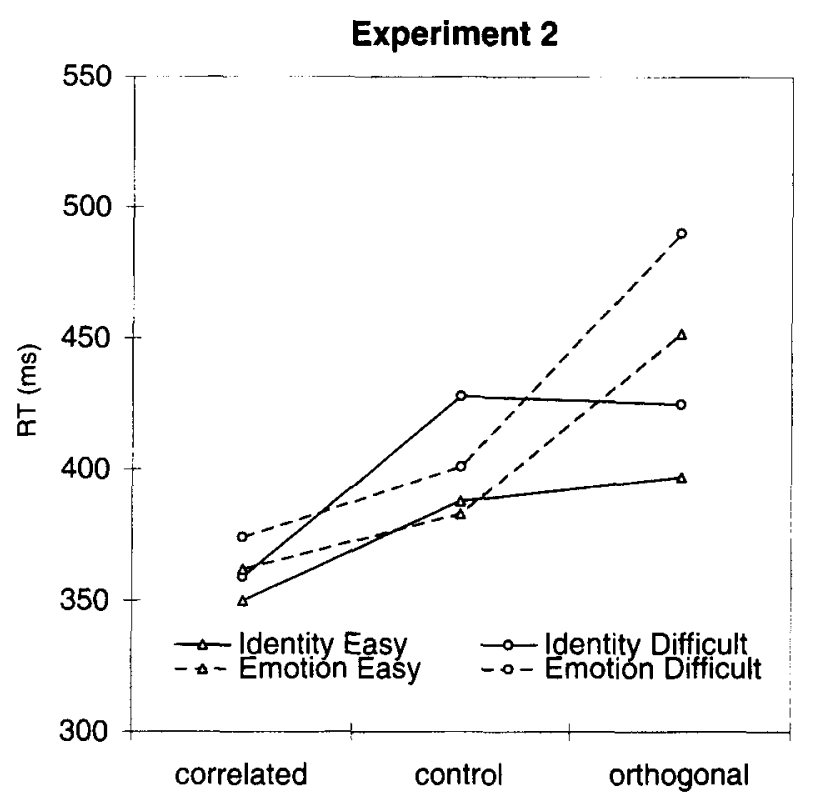

Experimental Condition

Figure 5. Mean reaction times in Experiments 2A and 2B for each combination of condition and difficulty.

was a significant main effect of condition $[F(2,44)=$ $58.2, p<.001]$ and a significant interaction of condition $\times$ experiment $[F(2,44)=13.1, p<.001]$. In addition, there was a highly significant effect of difficulty $[F(1,22)=$ $106.6, p<.001]$. There were also significant interactions of difficulty $\times$ condition $[F(2,44)=18.5, p<.001]$ and of difficulty $\times$ condition $\times$ experiment $[F(2,44)=9.7$, $p<.001]$.

To further analyze these interactions, we subsequently performed separate ANOVAs for each experiment, regarding as variables the condition, the particular alternative of the relevant dimension, and the particular alternative of the irrelevant dimension as described earlier. If there were any significant effects of condition in these analyses, Bonferroni-corrected comparisons were performed for the correlated versus control conditions, and for the orthogonal versus control conditions.

Experiment 2A. The analysis of the RTs revealed a significant effect of condition $[F(2,22)=24.6, p<.001]$, which was due to a redundancy gain $[F(1,11)=45.6, p<$ $.001]$, with no difference between the orthogonal and the control conditions $[F<1, M=355,408$, and $411 \mathrm{msec}$ for the correlated, control, and orthogonal conditions, respectively]. There was also a significant effect of difficulty $[F(1,11)=58.9, p<.001]$ and a significant interaction of condition $\times$ difficulty $[F(2,22)=14.8, p<.001]$. There were no significant effects or interactions involving the particular alternative of either the relevant or the irrelevant dimension.

In the comparison between the control and the orthogonal condition, there was of course a main effect of difficulty $[F(1,11)=86.6, p<.001]$. However, the interaction of difficulty $X$ condition was no longer significant. Note that the numerical effect of the orthogonal condition was, if anything, smaller for difficult than for easy stimuli ( $M=-3 \mathrm{msec}$ vs. $10 \mathrm{msec}$ for difficult vs. easy stimuli; see Figure 5). This clearly shows that there is no influence of irrelevant variations in emotional expression on identity classifications, even when the identity dimension is more difficult to perceive than the emotion dimension. In the comparison between the correlated and the control condition, the main effect of difficulty $[F(1,11)=48.7$, $p<.001]$ and the interaction of difficulty $\times$ condition $[F(1,11)=22.4, p<.001]$ were retained. This interaction reflected the fact that the effect of difficulty was much reduced in the correlated as compared with the control condition ( $M=10 \mathrm{msec}$ vs. $41 \mathrm{msec}$; see Figure 5).

Experiment 2B. The analysis of the RTs yielded a significant effect of condition $[F(2,22)=42.2, p<$ $.001]$, which was due to a redundancy gain $[F(1,11)=$ $10.1, p<.01]$, as well as a large difference between the orthogonal and the control conditions $[F(1,11)=35.1$, $p<.001 ; M=368,392$, and $471 \mathrm{msec}$ for the correlated, control, and orthogonal conditions, respectively]. There was also a significant effect of difficulty $[F(1,11)=24$. $p<.001]$ and a significant interaction between condition and difficulty $[F(2,22)=13.3, p<.001]$. There was a significant main effect of the particular alternative of the relevant dimension $[F(1,11)=24.1, p<.001]$, reflecting the fact that responses to angry faces were faster than those to happy faces ( $M=397$ vs. $424 \mathrm{msec}$ ). The only other significant effect was a complex four-way interaction [condition $\times$ difficulty $\times$ relevant $\times$ irrelevant dimension, $F(2,22)=6.6, p<.01]$.

In the comparison between the control and the orthogonal conditions, there was a main effect of difficulty $[F(1,11)=45.9, p<.001]$ and a significant interaction of difficulty $\times$ condition $[F(1,11)=13.4, p<.01]$. The interaction indicates that the interference seen in the orthogonal condition was somewhat larger for difficult than for easy stimuli ( $M=89 \mathrm{msec}$ vs. $69 \mathrm{msec}$; see Figure 5). The four-way interaction mentioned above was not significant in this comparison $[F(2,22)=1.26, p>.20]$. In the comparison between the correlated and the control conditions, there was a main effect of difficulty $[F(1,11)=$ $20.6, p<.001]$, but no interaction of difficulty $\times$ condition $[F(1,11)=1.5, p>.20]$. The effect of difficulty was slightly but not significantly reduced in the correlated as compared with the control condition ( $M=10 \mathrm{msec}$ vs. $18 \mathrm{msec}$; see Figure 5). The four-way interaction mentioned above was significant $[F(1,11)=8.1, p<.05]$. Inspection of Figure 4 suggests that for easy stimuli, somewhat larger redundancy gains were observed for the person B happy and the person A angry faces, whereas for difficult stimuli, somewhat larger redundancy gains were observed for the person A happy and the person B angry faces. Although this four-way interaction is difficult to account for, it seems to be weak relative to the other effects observed in this experiment. Also, it is superimposed on a consistent overall pattern, and it is confined 
to the comparison between the correlated and control conditions. We will therefore not discuss this interaction further.

\section{Discussion}

In this experiment, we explored the relationship between the perception of facial identity and emotion in a speeded classification task that required the participants selectively to attend to one dimension. We reasoned that if one dimension (e.g., identity) can be perceived independently of a second dimension (e.g., emotion), classification RTs for the first dimension should be unaffected by taskirrelevant variations in the second dimension. The results show that identity classifications were unaffected by irrelevant variations in facial emotion, whereas emotion classifications were slowed by irrelevant variations in facial identity. We reasoned further that if an insensitivity of identity classifications for emotion variation is a result of the higher perceptual speed of identity, identity classifications should be affected by irrelevant emotion variations if identity information is perceived more slowly than emotion information. However, the results show that even when task-relevant identity information was more difficult and was discriminated more slowly than taskirrelevant emotion information, identity classifications were still independent of irrelevant emotion information. Therefore, the present pattern of asymmetric relationship is not a result of different discriminabilities of identity and emotion.

The asymmetric relationship also shows that the difference between the control and the orthogonal conditions in the emotion classification task is not simply a result of the fact that there were four different stimuli in the control condition and eight different stimuli in the orthogonal condition. If this were the case, there should have been an RT increase in the orthogonal condition for both the emotion and the identity classification tasks (see also Green, Tomiak, \& Kuhl, 1997).

The results from the correlated condition show that there was some degree of redundancy gain in all conditions of the experiment. This is in contrast to the results obtained by Schweinberger and Soukup (1998, Experiment 1 ), who found no redundancy gain when identity was the relevant dimension. These differences may be explained by the fact that the relevant dimension was, on the average, less perceptually salient than the irrelevant dimension in the present study. Consequently, attending to the irrelevant dimension despite the task instruction would have been more advantageous for performance in the correlated condition of the present study, compared with the correlated condition of the Schweinberger and Soukup study. In this context, it is important to note that for identity classifications (Experiment $2 \mathrm{~A}$ ), the difficulty effect was greatly reduced in the correlated condition specifically. This is in line with the idea that, particularly when identity was difficult to perceive, participants shifted their attention to the irrelevant but more salient emotion dimension in that condition.

\section{GENERAL DISCUSSION}

In the first experiment of this paper, it was demonstrated that images on a continuum between two identities or emotional expressions of faces are consistently classified into one of the two categories over a broad range of the continua. The classification RTs did not show a general effect of morphing; only when images were morphed across the classified dimension did RTs reflect an increased difficulty in classifying images that were further away from the endpoints. This suggests that morphing can be used to manipulate selectively the perceptibility of either the identity or the expression of faces. In the second experiment, a subset of these stimuli was employed in order to investigate the role of perceptual speed in the relationship between identity and expression perception. In a Garner-type speeded classification task, irrelevant variation in facial identity clearly interfered with the perception of emotion. At the same time, the perception of facial identity was found to be independent of irrelevant variation in facial emotion. This asymmetric relationship was not related to a faster perceptibility of the identity variation in the stimuli: Even when identity was more difficult to discriminate than emotion, the asymmetric relationship was maintained. This pattern of results replicates and extends the previous findings of Schweinberger and Soukup (1998).

What could be the underlying reasons for an asymmetric relationship between the perception of identity and expression? First, identity might be a more "basic" type of information. As a result of interindividual variability in the expression of emotion, taking identity into account might provide a reference with respect to which the more transient changes - such as those seen in emotions-may be more easily processed by the perceptual system. A second possibility might be to consider the types of variation in faces that convey identity and expression, respectively. Emotional expressions may be regarded mainly as spatial transformations of one and the same face. In contrast, it appears that certain types of nonspatial variation, such as texture and information about areas of relative light and dark, are much more important for recognizing facial identity (see, e.g., Bruce, Hanna, Dench, Healey, $\&$ Burton, 1992). It is not clear whether the present findings are related to some special status of identity, or to the specific stimulus variations that happen to convey identity information in faces. One way to decide between these possibilities, however, would be to consider studies with speech stimuli that, like faces, could also be analyzed for identity, emotion, or speech content. If the analysis of identity has a privileged status, a similar pattern of asymmetric interference would be expected for speech stimuli. In contrast, there would be no reason to expect a similar pattern for speech stimuli if the critical factor for the present results was the stimulus variations that happen to convey identity information in faces.

To the best of our knowledge, there are no comparable studies on the relationship between identity and emo- 
tion perception in spoken language. Of particular interest, however, are studies on the relationship between phonetic information and talker information in speech perception, which also tend to reveal an asymmetric pattern of interference: When listeners are required to perform classifications for voice gender, there are few if any effects of irrelevant variations in phonetic information, as compared with clear effects of talker variations on classifications of phonetic content (Green et al., 1997; Mullenix \& Pisoni, 1990). This finding is reminiscent of previous results concerning the relationship between facial identity and facial speech perception (Schweinberger \& Soukup, 1998, Experiments 3-7), and it is consistent with a growing literature on the effects of talker variability in speech processing ( for an overview, see Johnson \& Mullenix, 1997). One implication of such findings might be that identity information in faces and voices has a special status, in that it is taken into account by the systems that compute other types of social information, such as speech or emotional expression. However, it clearly remains for future studies to provide a more direct comparison of results from the visual and auditory modalities.

Recently, it has been suggested that different expressions of basic emotions may have evolved quite separately, so that their perception might be linked to different neural substrates. For example, there is some evidence for a specific involvement of the amygdala in the perception of fear (Calder, Young, Rowland, et al., 1996), and of the basal ganglia in the perception of disgust (Sprengelmeyer et al., 1996). It may therefore be asked whether the pattern of asymmetric relationship found in the present study is specific to the basic emotions (happiness and anger) that were investigated here. However, we have found basically the same pattern with all the emotions that we have investigated thus far-happiness, anger, fear, and sadness (cf. Schweinberger et al., 1998, Schweinberger \& Soukup, 1998). Although the issue of specialized mechanisms for particular emotions clearly deserves further attention, it appears at present that our results do generalize across different basic emotions.

In spite of these comments, one needs to note that we have only studied two alternatives of each stimulus dimension at a time, and, for a given subject, there were only eight different stimuli in Experiment 2. A potential issue could therefore be that stimulus-based strategies (e.g., attending to a particular feature such as a bigger nose or bigger eyebrows of person A) may have played a role in producing the present findings of asymmetric relationships. For now, we have two reasons to believe that stimulusbased strategies cannot explain our findings. First, any discrimination between faces on the basis of a single feature should also have been affected by our difficulty manipulation. This is because the morphing procedure reduces not only the overall discriminability between faces but also the discriminability of each single facial feature. Yet the overall pattern of asymmetric interference was similar for all experimental conditions. In this respect, it is also interesting that Mullenix and Pisoni (1990), who used an auditory selective attention paradigm, systematically, varied the number of stimuli in both dimensions between $2 \times 2$ and $16 \times 16$. Stimulus-based strategies, it there are any, should become progressively unlikely as the number of stimuli is increased. Although Mullenix and Pisoni found some increase in overall orthogonal interference with more stimuli, the degree of asymmetric interference was unaffected by the number of stimuli. Even though we therefore think that stimulus-based strategies probably cannot account for the present findings, we plan to investigate more systematically the effects of additional pictorial variations in future studies.

What type of processing model can account for the asymmetric pattern of results obtained in the present study? The data clearly pose difficulties for a model of parallel and independent processes for identity and expression perception (Bruce \& Young, 1986). Such a model would predict that there is a mutual independence of identity and emotion, in which irrelevant variation on one dimension does not interfere with the perception of the other dimension. In contrast to this prediction, however, we found that identity interferes with the perception of facial emotion. Another possibility would be to assume a serial model, in which identity is encoded at an early level and expression is encoded only at a later level. However, a simple serial model has difficulty in explaining why, for certain stimuli, emotional expressions can actually be perceived faster than identity, and why the same pattern of asymmetric interference can be found even under these conditions. A third possibility, one that is consistent with the present findings, is that facial identity and emotion information are encoded in a parallel-contingent fashion (for a discussion of these types of models, see also Green et al., 1997; Mullenix \& Pisoni, 1990). Thus, the computation of facial identity and emotion may be performed in parallel, but emotion perception may be moderated by the output of identity perception, whereas identity perception can be carried out independently of the output of emotion perception.

In conclusion, Experiment 2 of the present study confirms an asymmetric relationship between the perception of facial identity and facial emotion, and the data suggest that this asymmetric relationship is not a result of differences between identity and emotion in terms of perceptibility. However, it might be worth noting that, whereas Experiment 2 revealed a pattern of asymmetric interference between identity and expression perception, certain aspects in the results of Experiment 1 would appear to be more consistent with previous ideas of an independence of identity and expression perception. Specifically, identity classifications did not seem to be much affected by whether or not these faces showed a clear-cut emotional expression; the functions were flat for faces morphed across emotional expressions. Conversely, emotion classifications did not seem to be much affected by whether or not these faces were morphed across identity. This could suggest that there may be theoretically important limitations for an influence of identity on emotion perception. 
One way to account for the present findings would be to adopt a perceptual "tuning" account. Accordingly, identity information for a given facial pattern could be computed and held in working memory, in order to provide a reference for computing information about facial expressions. When facial identity varies from trial to trial in the orthogonal condition, facial identity needs to be recomputed, which takes time and produces the interference effect seen in the orthogonal condition in Experiment 2 . This account would imply that the influence of identity on expression perception should be entirely contingent on a trial-to-trial variation of facial identity. On the one hand, such an explanation would be consistent with the results of both experiments in the present paper. It may also be interesting to mention recent auditory studies which have suggested that an influence of voice identity on speech perception is largely due to a trial-to-trial variation of voice identity (Green et al., 1997). On the other hand, previous research has also demonstrated a facilitative effect of personal familiarity with a face on the perception of expressive speech patterns in the face (Schweinberger \& Soukup, 1998, Experiment 5). This does not suggest that an influence of identity on the perception of expressive changes in the face is confined entirely to trial-to-trial variations in identity. A challenge for future studies will be to delineate more clearly the conditions in which interactions between expression and identity information occur in face perception.

\section{REFERENCES}

Beale, J. M., \& Keil, F. C. (1995). Categorical effects in the perception of faces. Cognition, 57, 217-239.

BRUCE, V. (1986). Influences of familiarity on the processing of faces. Perception, 15, 387-397.

Bruce, V., Hanna, E., Dench, N., Healy, P., \& Burton, A. M. (1992). The importance of "mass" in line drawings of faces. Applied Cognitive Psychology, 6, 619-628.

BRUCE, V., \& Young, A. (1986). Understanding face recognition. British Journal of Psychology, 77, 305-327.

Calder, A. J., Young, A. W., Perrett, D. I., Etcoff, N. L., \& RowLAND, D. (1996). Categorical perception of morphed facial expressions. Visual Cognition, 3, 81-117.

Calder, A. J., Young, A. W., Rowland, D., Perrett, D. I., Hodges, J. R., \& ETCOFF, N. L. (1996). Facial emotion recognition after bilateral amygdala damage: Differentially severe impairment of fear. Cognitive Neuropsychology, 13, 699-745.

Campbell, R., De Gelder, B., \& De HaAn, E. H. F. (1996). The lateralization of lip-reading: A second look. Neuropsychologia, 34, 1235-1240.

De Renzi, E., Faglioni, P., \& Spinnler, H. (1968). The performance of patients with unilateral brain damage on face recognition tasks. Cortex, 4, 17-34.

Eimas, P. D., Tarteer, V. C., Miller, J. L., \& Keuthen, N. J. (1978). Asymmetric dependencies in processing phonetic features. Perception \& Psychophysics, 23, 12-20.

ELLIS, A. W. (1989). Neuro-cognitive processing of faces and voices. In A. W. Young \& H. D. Ellis (Eds.), Handbook of research on face processing (pp. 207-215). Amsterdam: North-Holland.

Ellison, J. W., \& Massaro, D. W. (1997). Featural evaluation, integration, and judgment of facial affect. Journal of Experimental Psychology: Human Perception \& Performance, 23, 213-226.
ETcoFf, N. L. (1984). Selective attention to facial identity and facial emotion. Neuropsychologia, 22, 281-295.

Etcoff, N. L., \& MAGEE, J. J. (1992). Categorical perception of facial expressions. Cognition, 44, 227-240.

GARNER, W. R. (1974). The processing of information and structure. Potomac, MD: Lawrence Erlbaum.

GARNER, W. R. (1983). Asymmetric interactions of stimulus dimensions in perceptual information processing. In T. J. Tighe \& B. E. Shepp (Eds.), Perception, cognition, and development: Interactional analyses (pp. 1-38). Hillsdale, NJ: Erlbaum.

GreEN, K. P., \& KUHL, P. K. (1991). Integral processing of visual place and auditory voicing information during phonetic perception. Journal of Experimental Psychology: Human Perception \& Performance, 17, 278-288.

Green, K. P., Tomiak, G. R., \& Kuhl, P. K. (1997). The encoding of rate and talker information during phonetic perception. Perception \& Psychophysics, 59, 675-692.

Hasselmo, M. E., Rolls, E. T., \& Baylis, G. C. (1989). The role of expression and identity in the face-selective responses of neurons in the temporal visual cortex of the monkey. Behavioural Brain Research, 32, 203-218.

Humphreys, G. W., Donnelly, N., \& Riddoch, M. J. (1993). Expression is computed separately from facial identity, and is computed separately for moving and static faces: Neuropsychological evidence. Neuropsychologia, 31, 173-181.

HuYNH, H., \& FELDT, L. S. (1976). Estimation of the box correction for degrees of freedom from sample data in randomized block and split plot designs. Journal of Educational Statistics, 1, 69-82.

JoHnson, K., \& MULLENIx, J. W. (1997). Talker variability in speech processing. San Diego: Academic Press.

MCGurK, H., \& MACDONALD, J. (1976). Hearing lips and seeing voices. Nature, 264, 746-748.

Mullenix, J. W., \& Pisoni, D. B. (1990). Stimulus variability and processing dependencies in speech perception. Perception \& Psychophysics, 47, 379-390.

Schweinberger, S. R., Burton, A. M., Elbert, T., Junghöfer, M., Kelly, S. W., Müller, M. M., \& Soukup, G. R. (i998). Wechselwirkungen zwischen der Wahrnehmung von Identität und Emotion in Gesichtern [Interactions between perception of identity and emotion in faces]. In H. Lachnit, A. Jacobs \& F. Rösler (Eds.), Experimentelle Psychologie (pp. 333-334). Lengerich, Germany: Pabst Science Publishers.

SCHWeinberger, S. R., \& SoukuP, G. R. (1998). Asymmetric relationships among perceptions of facial identity, emotion, and facial speech. Journal of Experimental Psychology: Human Perception \& Performance, 24, 1748-1765.

SERGENT, J. (1985). Influence of task and input factors on hemispheric involvement in face processing. Journal of Experimental Psvchology: Human Perception \& Performance, 11, 846-861.

Sergent, J., Ohta, S., MacDonald, B., \& Zuck, E. (1994). Segregated processing of facial identity and emotion in the human brain: A PET study. Visual Cognition, 1, 349-369.

Sprengelmeyer, R., Young, A. W., Calder, A. J., Karnat, A., Lange, H. W., Hömberg, V., Perrett, D. I., \& Rowland, D. (1996). Loss of disgust: Perception of faces and emotions in Huntington's disease. Brain, 119, 1647-1665.

Uttal, W. R., Baruch, T., \& Allen, L. (1995). The effect of combinations of image degradations in a discrimination task. Perception \& Psychophysics, 57, 668-681.

Walker, S., Bruce, V., \& O'Malley, C. (1995). Facial identity and facial speech processing: Familiar faces and voices in the McGurk effect. Perception \& Psychophysics, 57, 1124-1133.

Young, A. W., McWeeny, K. H., HaY, D. C., \& Ellis, A. W. (1986). Matching familiar and unfamiliar faces on identity and expression. Psychological Research, 48, 63-68.

Young, A. W., Rowland, D., Calder, A. J., Etcoff, N. L., Seth, A., \& Perrett, D. I. (1997). Facial expression megamix: Tests of dimensional and category accounts of emotion recognition. Cognition, 63, 271-313. 


\section{NOTES}

1. In previous studies, researchers have tried to deal with this problem by excluding the original stimuli from their material, making sure that all stimuli underwent the morphing procedure. For instance, Beale and Keil (1995) used the $1 \%$ and $99 \%$ morphed images rather than the original ones, and Young et al. (1997; but see also Calder, Young, Perrett, et al., 1996) used the $10 \%$ and $90 \%$ images. Nevertheless, this may not be a satisfactory precaution against general effects of the morphing process per se, because such effects would critically depend on the distance of an image from the endpoints. In the present study, we decided to use the original images along with the morphed versions.

2. Because the spatial transformation of the control points in warping is linear, as is the transformation of pixel identities in fading, it has sometimes been claimed that morphing produces a linear continuum between two stimuli. This is not without problems, however, since the measure- ment scale in morphed stimuli is some combination of the 2-D shape of the face and the pixel intensities.

3. A sharp transition point is sometimes taken as evidence for categorical perception (usually in combination with the demonstration that the perceptual discriminability between two equidistant stimuli is increased when the two stimuli cross the boundary between both categories, relative to when they vary within a category. However, the shape of the classification function alone cannot be regarded as unambiguous evidence for categorical perception (see, e.g., Calder, Young, Perrett, et al., 1996; Ellison \& Massaro, 1997). Because our intention in the present paper is not to argue for or against categorical perception, we will not elaborate on this issue.

(Manuscript received February 24, 1998; revision accepted for publication August 31, 1998.) 